\section{Anti-C5 antibody treatment for delayed hemolytic transfusion reactions in sickle cell disease}

Delayed hemolytic transfusion reaction (DHTR) is an unpredictable severe complication of transfusion in patients with sickle cell disease (SCD). It presents clinically as a vaso-occlusive crisis (VOC), often associated with the failure of one or more organs, after the transfusion of packed red blood cells (pRBC) ${ }^{1,2}$ Hyperhemolysis is encountered in the most severe forms. Both transfused and autologous red blood cells (RBC) are lysed.

The mechanisms underlying DHTR remain unclear.
Alloantibodies against RBC antigens were initially thought to underlie the pathophysiology, but no such antibodies are detected in about a third of the cases. ${ }^{3}$

RBC degradation products, such as hemoglobin and heme, are released into the bloodstream during intravascular hemolysis. These elements and heme-loaded membrane microvesicles have recently been implicated in inflammation and organ injury in DHTR. ${ }^{4}$ Complement is activated via the classical pathway, by alloantibodies, and/or via the alternative pathway, by free heme. ${ }^{5} \mathrm{Heme}-$ dependent complement deposits on the endothelium contribute to organ damage. ${ }^{6}$ Due to these vascular lesions, hyperhemolysis often progresses to multiple

Table 1. Clinical and biological findings at diagnosis and during follow-up.

\begin{tabular}{|c|c|c|c|}
\hline . & This series & Habibi et al. & \\
\hline \multicolumn{4}{|l|}{ Patient characteristics } \\
\hline Number of patients; DHTR episodes & $18 ; 18$ & $69 ; 99$ & \\
\hline $\mathrm{Hb} \beta^{\mathrm{s}} \beta^{\mathrm{s}}$ & $18(100 \%)$ & $65(94.2 \%)$ & \\
\hline Sex F/M & $11 / 7$ & $48 / 21$ & \\
\hline Age, years & $24.6 \pm 12.6$ & $30 \pm 9$ & \\
\hline Number of pRBC units in transfusion episode & $2 \pm 1.9$ & $2 \pm 3$ & \\
\hline \multicolumn{4}{|l|}{ Transfusion indications } \\
\hline Preventive measure & $5(27.8 \%)$ & $51(51.5 \%)$ & \\
\hline Vaso-occlusive complications & $11(61.1 \%)$ & $48(48.5 \%)$ & \\
\hline Other & $2(11.1 \%)$ & & \\
\hline \multicolumn{4}{|l|}{ Timeline } \\
\hline Days from transfusion to DHTR diagnosis & $8[7-12.8]$ & $10[8-14](\mathrm{MD}=19)$ & \\
\hline Days from transfusion to anti-C5 infusion & $10.5[9-15.5]$ & & \\
\hline \multicolumn{4}{|l|}{ Biological findings in the emergency room } \\
\hline Total Hb level, g/dL & $63.5[53.3-77.8]\left(\mathrm{NA}=6^{*}\right)$ & 78 [69-93] $(\mathrm{MD}=5)^{\dagger}$ & $P=0.03$ \\
\hline LDH level, IU/L & $1612[825-2702]\left(\mathrm{NA}=6^{*}\right)$ & $758[554-958](\mathrm{MD}=16)$ & $P<0.01$ \\
\hline \multicolumn{4}{|l|}{ Treatment** } \\
\hline EPO & $17(94.4 \%)$ & $45 \%$ & \\
\hline Corticosteroids & $1(5.6 \%)$ & $3 \%$ & \\
\hline Plasma or albumin exchange & $4(22.2 \%)$ & & \\
\hline IV immunoglobulins & $9(50 \%)$ & $4 \%$ & \\
\hline Anti-CD20 antibody & $7(38.9 \%)$ & $2 \%$ & \\
\hline Anti-C5 antibody & $16(100 \%)$ & $2 \%$ & \\
\hline Secondary pRBC transfusion & $14(77.8 \%)$ & $35 \%$ & \\
\hline \multicolumn{4}{|l|}{ Extreme biological findings } \\
\hline Lowest total Hb level, g/dL & $30.5[25.5-42.8]$ & $55[45-63](\mathrm{MD}=5)^{\dagger}$ & $P<0.01$ \\
\hline Highest LDH level, IU/L & $3337[2573-7986]$ & 1335 [798-2086] (MD=7) & $P<0.01$ \\
\hline Lowest reticulocyte count, $10^{9} / \mathrm{L}$ & $46.1[35.8-84.8](\mathrm{MD}=2)$ & 180 [121-240] (MD=14) & $P<0.01$ \\
\hline Delta $\mathrm{Hb}^{\ddagger}, \mathrm{g} / \mathrm{dL}$ & $57.5[45.8-67.5](\mathrm{MD}=4)$ & $46[31-53](\mathrm{MD}=26)^{\dagger}$ & $P=0.06$ \\
\hline \multicolumn{4}{|l|}{ Outcome } \\
\hline ICU admission & $17(94.4 \%)$ & $41(40 \%)$ & \\
\hline ICU-stay duration, days & $17.7 \pm 10.2$ & $6.2 \pm 4$ & $P<0.01$ \\
\hline Hospital-stay duration, days & $35.6 \pm 25.3$ & $15.9 \pm 10$ & $P<0.01$ \\
\hline Transfusion-to-death interval, days & $51.7 \pm 47.9$ & $10 \pm 2$ & \\
\hline Death & $3(16.7 \%)$ & $6 \%$ & \\
\hline
\end{tabular}




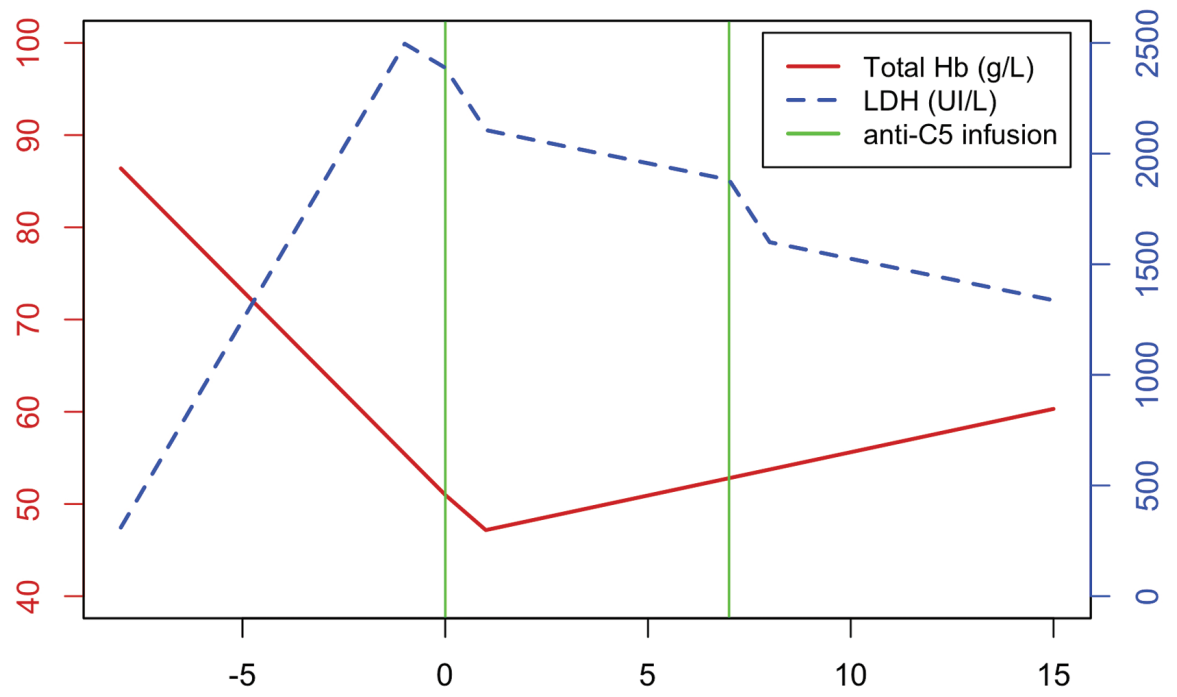

days after anti-C5 infusion

Figure 1. Best mixed-effects model for total hemoglobin and lactate dehydrogenase during delayed hemolytic transfusion reaction, before and after anti-C5 antibody infusion. Hemoglobin $(\mathrm{Hb})$ levels are predicted for a "theoretical" patient receiving anti-C5 antibody infusions on days 0 and 7 and no packed red blood cell (pRBC) transfusions. Before anti-C5 antibody infusion, basal total $\mathrm{Hb}$ concentration in this model was $51.0 \mathrm{~g} / \mathrm{L}$ (the intercept of the model), with an increase of $+3.8 \mathrm{~g} / \mathrm{L}$ for each pRBC unit transfused, and ongoing hemolysis at a rate of $-4.4 \mathrm{~g} / \mathrm{L}$ for each passing day (the fixed effects of the model). However, after anti$\mathrm{C} 5$ antibody infusion, $\mathrm{Hb}$ levels gradually increased, with a basal $\mathrm{Hb}$ concentration of $46.2 \mathrm{~g} / \mathrm{L}$ : the effect of each transfusion was an increase of $1.58 \mathrm{~g} / \mathrm{L}$ for each pRBC unit transfused and an increase in $\mathrm{Hb}$ levels of $0.94 \mathrm{~g} / \mathrm{L}$.

organ failure and, in some cases, death.

These pathophysiological findings suggest that inhibitors of complement activation may be useful for treating DHTR with hyperhemolysis. Eculizumab is a monoclonal anti-C5 antibody that inhibits the cleavage of $\mathrm{C} 5$ into $\mathrm{C} 5 \mathrm{a}$ by the $\mathrm{C} 5$ convertase, thereby preventing the late stages of the complement cascade. Anti-C5 therapy has been offered to several SCD patients for DHTR treatment at our SCD referral center in France since 2013. ${ }^{1,7}$ Other teams have also treated DHTR in patients with and without detectable allo- or auto-antibody formation with Eculizumab, with promising results. ${ }^{8-11}$ The American Society of Hematology (ASH) guidelines include a conditional recommendation for the use of antiC5 antibodies in patients with SCD presenting DHTR and ongoing hyperhemolysis, based on currently very low levels of certainty. ${ }^{12}$

This retrospective study focuses on the biological and clinical findings and the effects of anti-C5 therapy on DHTR, for patients treated between 2013 and 2019 who experienced particularly severe DHTR.

DHTR was diagnosed ${ }^{1,2}$ on the basis of VOC signs occurring 5-20 days after pRBC transfusion, with no other identifiable cause of intravascular hemolysis, in association with at least one of the following signs:

- rapid decrease in, or unexpectedly low, hemoglobin A ( $\mathrm{HbA}$ ) concentration (the diagnostic nomogram for DHTR diagnosis was used), ${ }^{2}$

- hemoglobinuria, as revealed by dark urine,

- positive direct antiglobulin test (DAT) results or new antibody formation.

The criteria for the use of anti-C5 therapy was based either on the existence, at the time anti-C5 infusion was decided, of one or more organs with dysfunction and/or very low total $\mathrm{Hb}$ concentration $(<50 \mathrm{~g} / \mathrm{L})$, and/or a rapidly worsening clinical state.

Data were collected retrospectively from patient records. The clinical and biological findings available at transfusion, at the time of DHTR diagnosis and during follow-up were collected. We also noted patient sex, age, history of DHTR, pRBC transfusion, and antibody screens. We recorded the number of $\mathrm{pRBC}$ units and the indication of the transfusion(s) occurring within a timeframe compatible with DHTR (some patients had received $\mathrm{pRBC}$ on several occasions during the 5-20 days preceding DHTR). Clinical (hemoglobinuria, pain and VOC signs, organ failure) and biological (hemoglobin concentration, reticulocyte count, LDH, total bilirubin) findings at DHTR diagnosis were collected. The first clinical signs compatible with DHTR were noted, particularly pain indicative of VOC recurrence, and hemoglobinuria indicative of intravascular hemolysis. We collected follow-up data for biological tests, intensive care unit admission and discharge, organ failure and treatments. This study was performed in accordance with the Declaration of Helsinki.

We used R3.6.1 and lme $4^{13}$ for a linear mixed-effects model analysis of the relationship between $\mathrm{Hb}$ and lactate dehydrogenase (LDH) levels and anti-C5 treatment. $\mathrm{Hb}$ and $\mathrm{LDH}$ levels were modeled before and after treatment. An initial blind statistical analysis was performed, and several models were then proposed, with days and $\mathrm{pRBC}$ transfusions as fixed effects, and different combinations of random effects for subjects, days and pRBC transfusions. We obtained $P$-values for likelihood ratio tests of the full model with random effects against the model without additional terms, which we used to select the best model.

Eighteen SCD patients received anti-C5 treatment for DHTR with hyperhemolysis. All patients had signs of VOC 5-20 days after pRBC transfusion, with low $\mathrm{HbA}$ concentrations $(<10 \mathrm{~g} / \mathrm{L})$ in five patients, a rapid decrease in $\mathrm{HbA}$ concentration in 10 patients (estimated by the nomogram ${ }^{2}$ as a high $(n=4)$ or intermediate $(n=6)$ risk of 
DHTR), hemoglobinuria in 11 patients, and positive DAT results or antibody formation in 12 patients (anti-MNS3, anti-KEL6, anti-RH10 + anti-RH20, anti-MNS5, anti-FY5 in one patient each, one patient developed multiple antibodies including anti-MNS3, anti-RH20 and auto-antibodies, two patients developed auto-antibodies and two patients delevopde allo-antibodies for which the specificity could not be determined and two patients had positive DAT but no new antibody was subsequently identified). A $19^{\text {th }}$ patient received anti-C5 antibody for hyperhemolysis but it was impossible to determine whether this patient had DHTR due to hemolysis under extracorporeal membrane oxygenation, ${ }^{14}$ so this patient was excluded from the analysis.

The main characteristics of the patients are presented in Table 1. Sixteen patients (89\%) had risk factors for DHTR: a history of previous DHTR $(n=2)$, a history of RBC antibodies $(n=11)$, or the administration of fewer than $12 \mathrm{pRBC}$ units before the episode leading to DHTR $(n=11) .15$ Three patients had a history of ineffective pRBC transfusions, possibly due to previous undetected episodes of DHTR. None of the patients were enrolled in chronic transfusion programs. Five patients underwent repeat transfusions before the diagnosis of DHTR, which may have worsened their clinical presentation at diagnosis.

The findings at diagnosis and during follow-up, compared with those of a historical cohort ${ }^{1}$ are presented in Table 1 (see Online Supplementary Data for individual timelines). At diagnosis, the patients had particularly severe DHTR, with parameters highly indicative of hemolysis (low $\mathrm{Hb}$, high $\mathrm{LDH}$ concentrations), and the failure of at least one organ in $50 \%$ of cases $(n=9)$ : kidney failure $(n=7)$, liver failure $(n=4$, including two with indications for liver transplantation), respiratory failure $(n=5)$. Five patients had hemodynamic failure requiring treatment with vasoactive agents.

One to three anti-C5 doses were administered at 1week intervals (one dose $n=6$, two doses $n=9$ and three doses $n=1$ ), in association with other treatments (Table 1). Unfortunately, complement activation measurements were not performed for most patients. The number of $\mathrm{pRBC}$ units transfused was restricted as much as possible, to limit exacerbations of hyperhemolysis.

Remarkably, a worsening of clinical conditions during follow-up occurred only in the hours immediately following anti-C5 infusion (i.e., due to the progression of pre-existing organ damage due to DHTR; $n=2$ ), or as a result of sepsis due to additional infectious complications $(n=2)$. One patient suffered hemodynamic failure within a few hours of anti-C5 infusion. One patient (16P) with kidney failure, hemodynamic failure and a severe hepatic alteration before anti-C5 infusion rapidly progressed to hepatic failure a few hours after the first infusion.

The outcome was favorable in 15 patients (83\%), with a complete recovery of all failing organs. Three patients died $(17 \%)$. All three had acute liver failure requiring emergency transplantation (already present at DHTR diagnosis in two of these patients). Two patients improved after one and two anti-C5 infusions and were able to undergo transplantation. However, both died from infectious complications due to encapsulated bacteria unrelated to anti-C5 treatment but promoted by the immunosuppressive regimen: ventilator-associated pneumonia 11 days after transplantation in patient $8 \mathrm{H}$, and digestive and urinary infection 47 days after transplantation in patient $16 \mathrm{P}$. No compatible organ could be found for patient $3 \mathrm{C}$, who died one day after anti-C5 antibody infusion.
Despite the heterogeneity of the data, linear mixedeffect model analysis with adjustment to produce the best model $(P<0.05)$ highlighted an influence of the anti$\mathrm{C} 5$ antibody treatment on total $\mathrm{Hb}$ and $\mathrm{LDH}$ levels (Figure 1). The inversion of the slope for total $\mathrm{Hb}$ and $\mathrm{LDH}$ levels before and after anti-C5 treatment indicated that hyperhemolysis was stopped, or at least greatly decreased, by treatment. The gradual increase in $\mathrm{Hb}$ levels may also be due to the other treatments received by the patients, especially erythropoietin (EPO) (Table 1). The stimulation of erythropoiesis improves the reticulocyte count, and proportionally increases hemoglobin $S$ $(\mathrm{HbS})$. In several patients who received secondary RBC transfusion, $\mathrm{HbA}$ concentration was maintained post transfusion (e.g., patients $2 \mathrm{~B}, 15 \mathrm{O}, 16 \mathrm{P}, 17 \mathrm{Q}$ ).

In conclusion, this is the largest series to date of cases of severe DHTR with hyperhemolysis in SCD patients, treated with anti-C5 antibody. It demonstrates the effect of anti-C5 therapy against hyperhemolysis in DHTR, with remarkable beneficial effects on pre-existing organ failure and additional organ failure once the effects of the treatment are established. These findings consolidate the recommendation in the ASH guidelines to use anti-C5 antibody in patients with SCD and ongoing hyperhemolysis. ${ }^{12}$ Other anti-complement drugs may also be useful for treatment in this context. A prospective clinical trial would be required to determine whether all DHTR patients would benefit from anti-C5 therapy or whether such treatment is beneficial only for the most severe clinical presentations.

Aline Floch, ${ }^{1}$ Alexandre Morel, ${ }^{2}$ Fabian Zanchetta-Balint, ${ }^{2}$ Catherine Cordonnier-Jourdin, ${ }^{3}$ Slimane Allali, ${ }^{4}$ Maximilien Grall, ${ }^{5}$ Ghislaine Ithier, ${ }^{6}$ Benjamin Carpentier, Sadaf Pakdaman, Jean-Claude Merle, ${ }^{8}$ Radiviv Goulabchand," Tackwa Khalifeh, ${ }^{10}$ Ana Berceanu, ${ }^{11}$ Cécile Helmer, ${ }^{12}$ Christelle Chantalat-Auger, ${ }^{13}$ Véronique Frémeaux-Bacchi, ${ }^{14}$ Marc Michel, ${ }^{15}$ Mariane de Montalembert, ${ }^{4}$ Armand Mekontso-Dessap, ${ }^{16}$ France Pirenne, ${ }^{1}$ Anoosha Habibi ${ }^{2}$ and Pablo Bartolucci

'Etablissement francais du sang Ile de France, INSERM Unit 955 Laboratory of Excellence GR-Ex, Mondor Institute of Biomedical Research, Paris-Est Creteil University, Creteil; ${ }^{2}$ French Sickle Cell Referral Center, Henri Mondor Teaching Hospital, Assistance Publique-Hopitaux de Paris, Laboratory of Excellence GR-Ex, INSERM Unit 955, Mondor Institute of Biomedical Research, ParisEst Creteil University, Creteil; ${ }^{3}$ Department of Pharmacy, Henri Mondor Teaching Hospital, Assistance Publique-Hopitaux de Paris; ${ }^{4}$ Department of Pediatrics, Necker Hospital for Sick Children, Assistance Publique-Hopitaux de Paris, Laboratory of Excellence GREx, Paris Descartes University, Paris; ${ }^{5}$ Department of Internal Medicine, Rouen Teaching Hospital, Rouen; 'Hematology Unit, Reference Center of Sickle Cell Disease, Robert Debré Hospital, Assistance Publique-Hopitaux de Paris, Paris; 'Department of Hematology, Saint Vincent de Paul Hospital, Lille Catholic University, Lille; ${ }^{8}$ Department of Anesthesia and Surgical Intensive Care, Liver Intensive Care Unit, Henri Mondor Teaching Hospital, Assistance Publique-Hopitaux de Paris, Creteil; ' ${ }^{\circ}$ Department of Internal Medicine-Multiorganic Diseases, Local Referral Center for Autoimmune Diseases, Saint-Eloi Hospital, Montpellier University, Montpellier; ${ }^{10}$ Pediatric Medical-Surgical Department, Poitiers Teaching Hospital, Poitiers, France; "Intensive Care Hematology Unit, Besancon Teaching Hospital, Besancon; ${ }^{12}$ Etablissement francais du sang Auvergne-Rhône Alpes, Grenoble; ${ }^{13}$ Department of Internal Medicine, Bicetre Teaching Hospital, Assistance Publique-Hopitaux de Paris, Universite Paris 11, Le Kremlin-Bicetre, Paris; ${ }^{14}$ Laboratory of Immunology, European Georges Pompidou Hospital, Assistance Publique-Hopitaux de Paris, Mixed Health Research Unit INSERM 
872, Cordeliers Research Center, Paris; ${ }^{15}$ Department of Internal Medicine, National Referral Center for Immune Cytopenias, Henri Mondor Teaching Hospital, Assistance Publique-Hopitaux de Paris, Creteil and ${ }^{16}$ Medical Intensive Care Unit, Henri Mondor Teaching Hospital, Assistance Publique-Hopitaux de Paris, Creteil, France

Correspondence:

PABLOBARTOLUCCI - pablo.bartolucci@aphp.fer

Acknowledgments: the authors would like to thank Thierry Peyrard, CNRGS Institut National de la Transfusion Sanguine, Paris; Claire Boulat, Etablissement français du sang, Le Kremlin-Bicetre; Paris; Badrdine El Masmouhi, Etablissement français du sang, Poitiers; Maud Deray, Etablissement français du sang, Montpellier, for antibody screening data; Damien Oudin Doglioni and Myriam Maumy for their advice regarding the statistical analysis.

doi:10.3324/haematol.2020.253856

\section{References}

1. Habibi A, Mekontso-Dessap A, Guillaud C, et al. Delayed hemolytic transfusion reaction in adult sickle-cell disease: presentations, outcomes, and treatments of 99 referral center episodes. Am J Hematol. 2016;91(10):989-994.

2. Mekontso Dessap A, Pirenne F, Razazi K, et al. A diagnostic nomogram for delayed hemolytic transfusion reaction in sickle cell disease. Am J Hematol. 2016;91(12):1181-1184.

3. de Montalembert M, Dumont M-D, Heilbronner C, et al. Delayed hemolytic transfusion reaction in children with sickle cell disease. Haematologica. 2011;96(6):801-807.

4. Merle NS, Grunenwald A, Rajaratnam $\mathrm{H}$, et al. Intravascular hemolysis activates complement via cell-free heme and heme-loaded microvesicles. JCI Insight. 2018;3(12):96910.

5. Merle NS, Boudhabhay I, Leon J, Fremeaux-Bacchi V, Roumenina LT. Complement activation during intravascular hemolysis: Implication for sickle cell disease and hemolytic transfusion reactions. Transfus Clin Biol. 2019;26(2):116-124.

6. Merle NS, Paule R, Leon J, et al. P-selectin drives complement attack on endothelium during intravascular hemolysis in TLR-4/hemedependent manner. Proc Natl Acad Sci USA. 2019;116(13):62806285.

7. Pirenne F, Yazdanbakhsh K. How I safely transfuse patients with sickle-cell disease and manage delayed hemolytic transfusion reactions. Blood. 2018;131(25):2773-2781.

8. Dumas G, Habibi A, Onimus T, et al. Eculizumab salvage therapy for delayed hemolysis transfusion reaction in sickle cell disease patients. Blood. 2016;127(8):1062-1064.

9. Chonat S, Quarmyne M-O, Bennett CM, et al. Contribution of alternative complement pathway to delayed hemolytic transfusion reaction in sickle cell disease. Haematologica. 2018;103(10):e483-e485.

10. Unnikrishnan A, Pelletier JPR, Bari S, et al. Anti-N and anti-Doa immunoglobulin $\mathrm{G}$ alloantibody-mediated delayed hemolytic transfusion reaction with hyperhemolysis in sickle cell disease treated with eculizumab and HBOC-201: case report and review of the literature. Transfusion. 2019;59(6):1907-1910.

11. Vlachaki E, Gavriilaki E, Kafantari K, et al. Successful outcome of hyperhemolysis in sickle cell disease following multiple lines of treatment: the role of complement inhibition. Hemoglobin. 2018;42(56):339-341.

12. Chou ST, Alsawas M, Fasano RM, et al. American Society of Hematology 2020 guidelines for sickle cell disease: transfusion support. Blood Adv. 2020;4(2):327-355.

13. Bates D, Mächler M, Bolker B, Walker S. Fitting linear mixed-effects models using lme4. J Stat Softw. 2015;67(1):85262.

14. Boissier F, Bagate F, Schmidt M, et al. Extracorporeal life support for severe acute chest syndrome in adult sickle cell disease: a preliminary report. Crit Care Med. 2019;47(3):e26-e265.

15. Narbey D, Habibi A, Chadebech P, et al. Incidence and predictive score for delayed hemolytic transfusion reaction in adult patients with sickle cell disease. Am J Hematol. 2017;92(12):1340-1348. 